\title{
Arquitectura de Software para el Soporte de Comunidades Académicas Virtuales en Ambientes de Televisión Digital Interactiva
}

\author{
Wilmar Y. Campo, Gabriel E. Chanchí y José L. Arciniegas \\ Depto de Telemática, Fac. de Ingeniería Electrónica y Telecomunicaciones, Universidad del Cauca \\ Calle 5 No. 4-70, Popayán-Colombia, (e-mail: wilicampo, gabrielc, jlarci@unicauca.edu.co)
}

Recibido Sep. 25, 2012; Aceptado Oct. 16, 2012; Versión final recibida Dic. 14, 2012

\begin{abstract}
Resumen
En este artículo se propone y analiza una arquitectura de software para el soporte de comunidades académicas virtuales (CAV), en el contexto de la televisión digital interactiva. La arquitectura propuesta extiende los servicios hasta ahora definidos dentro del ambiente de e-learning, introduciendo conceptos y aplicaciones de la Web 2.0 mediante un esquema de consumo de servicios REST-JSON. Se describen los escenarios soportados por la arquitectura, los componentes y su interoperabilidad que permiten dotar de interactividad a los diferentes servicios en un entorno de televisión digital, y los avances de su implementación en el laboratorio. Además, se discuten dos servicios soportados y desplegados sobre la arquitectura: un tablón de mensajes y una sala de chat. Se muestra la capacidad de la arquitectura para soportar servicios flexibles pudiendo estos, estar asociados o no al contenido multimedia de la CAV. Esto permite una mejor gestión y uso eficiente del canal de retorno.
\end{abstract}

Palabras clave: arquitectura de software, comunidades académicas virtuales, televisión digital interactiva, e-learning

\section{Software Architecture for supporting Virtual Academic Communities in Interactive Digital Television Environments}

\begin{abstract}
In this paper software architecture scheme for supporting of virtual academic communities (CAV) in the context of the interactive digital television, is proposed and analyzed. The proposed architecture extends services defined until now within the e-learning environment, introducing concepts and applications of Web 2.0 through the consumption of the REST-JSON service scheme The scenarios supported by the architecture, the components and their interoperability that allow providing interactivity to different services in an environment of digital television, and the progress of its implementation in the laboratory, are described. In addition, two other services supported and deployed on the architecture are discussed: a message board and a chat room. The ability of the architecture to support flexible services, which can be associated or not to multimedia content of the CAV, is shown. This allows better management and efficient use of the return channel.
\end{abstract}

Keywords: software architecture, academic virtual community, interactive digital television, aprendizaje electrónico 


\section{INTRODUCCIÓN}

Internet ha tenido una marcada evolución en los últimos años, permitiendo el acceso y el intercambio de información de manera ágil, con características de flexibilidad en la tecnología de acceso y con capacidades de integración a nivel de servicios [Lago et al., 2010]. La tendencia actual de Internet es fomentar la colaboración y el trabajo en comunidad a través de servicios como: foros, wikis, blogs, entre otros, más conocidos como herramientas de la Web 2.0; estas herramientas están siendo integradas en plataformas tales como redes sociales, CAV, e-Learning o en servicios de alto nivel compuestos a partir de otros servicios [Ashwin, 2006, Schröder et al., 2010]. Por otra parte la Televisión Digital (TD) está llamada a garantizar el mismo o mayor grado de cubrimiento sobre la población que la televisión analógica, ya que es el siguiente paso en la evolución de esta última. Una de las principales características de la TD, es la capacidad para acceder a aplicaciones interactivas mediante un canal de retorno, razón por la cual se abre un amplio campo de posibilidades en cuanto a escenarios educativos que incluyan las ventajas de la TD y la Web 2.0. Es así como la TD está llamada a ser una de las tecnologías de la información y comunicación (TIC) que contribuyan dentro de la sociedad del conocimiento a reducir la brecha digital.

Por lo anteriormente expuesto, este artículo propone una arquitectura que responde a las necesidades de soporte de servicios para una CAV en ambientes de TDi, aprovechando las ventajas de las aplicaciones que han tenido éxito en Internet. Esta arquitectura da soporte a escenarios típicos de servicios usados en las CAV como mecanismo de apoyo a la educación virtual. Además, esta puede ser tomada como referencia para la implementación de servicios sobre la TDi en otros contextos (t-comercio, t-salud, t-gobierno, entre otros).La arquitectura planteada está basada en el esquema de consumo de servicios REST-JSON propuesto en Chanchí et al. [2011] el cual presenta cada servicio como un recurso, que puede ser accedido a través de peticiones reducidas HTTP. Cada petición es respondida a través de mensajes JSON, los cuales deben ser procesados por los clientes que consumen el servicio. Dadas las características del esquema usado, la arquitectura diseñada puede ser fácilmente extendida, teniendo en cuenta que cada servicio puede ser visto como parte de un puzle de aplicaciones. El modelo REST-JSON actualmente está siendo difundido para el despliegue de servicios en comunidades de Internet y redes sociales; así la presente arquitectura aporta un nexo entre el mundo de las Telecomunicaciones e Internet, permitiendo el acceso a aplicaciones de la Web 2.0 (para el soporte de CAV) en entornos de TDi.

Existen diferentes iniciativas a nivel mundial sobre temáticas relacionadas como el T-Learning (aprendizaje a través del la televisión) y la construcción de contenidos interactivos para televisión digital. El Proyecto EDiTV [Arciniegas et al., 2011], cuyo objetivo principal es: apoyar procesos educativos a distancia en el programa de Tecnología Agroindustrial, ofrecido por la Universidad del Cauca a través del uso de educación virtual, soportada en la combinación de tecnologías innovadoras de televisión interactiva con la infraestructura y, los servicios de la Red Nacional Académica de Tecnología avanzada-RENATA. Este proyecto logra establecer un laboratorio para el soporte de contenidos educativos a través de TDi, que constituye un paso previo para el desarrollo de plataformas que soporten CAV en ambientes de TDi, alcanzando resultados como los descritos por Campo et al. [2009, 2010a].

El Proyecto T-Maestro [Redondo et al., 2008] se basa en el desarrollo de las tecnologías de Televisión Digital-TD para emitir aplicaciones telemáticas interactivas junto con las señales audiovisuales, y su papel emergente en T-Learning y M-Learning (Aprendizaje mediante de dispositivos móviles). El objetivo de este proyecto es el diseño, desarrollo y validación de un entorno para la creación de propuestas formativas, válido en lo ámbitos de T-Learning y M-Learning, teniendo en cuenta las características técnicas y sociales que permiten superar los principales obstáculos detectados en las propuestas de e-Learning. El objetivo del proyecto es el diseño y desarrollo de un tutor inteligente denominado T-Maestro (Multimedia Adaptive Education SysTem based on Reassembling TV Objects), que incluye capacidades de adaptación y personalización en el marco del aprendizaje a través de TV, integrando contenidos lúdicos y educativos.

El proyecto BEACON [Sancin et al., 2009] orientado a la investigación sobre la Televisión Digital TerrestreTDT con tres objetivos fundamentales: Desarrollar interoperabilidad entre los estándares de la televisión digital terrestre europea y el brasileño, estudiar una metodología para el aprendizaje a distancia mediante televisión digital y ofrecer servicios de T-Learning para la inclusión social en Sao Paulo Brasil. El proyecto SOLITE, cuyo objetivo es el de aunar conocimiento de los grupos participantes mediante la puesta en común de su experiencia en la creación de sistemas y tecnologías de e-Learning, y su implementación en Software Libre. Un área de trabajo del proyecto SOLITE es T-Learning donde se estudian alternativas de solución de televisión digital interactiva para el contexto de la teleformación [Acevedo et al., 2010]. Proyecto ELU [Alic et al., 2008] dirigido a proveer un rango de facilidades que permitan a los profesionales crear de manera fácil sus cursos, en este proyecto la televisión es tenida en cuenta como el principal medio para desarrollar las capacidades de aprendizaje. El proyecto T-Learning [eLearning WORKSHOPS, 2012] es un proyecto cofinanciado por la Comunidad Europea y los Ministerios de Comunicación e Innovación 
Tecnológica italianos, cuyo objetivo es experimentar la TDi con fines formativos, incidiendo en los aspectos pedagógicos y tecnológicos, a través de la plataforma eXact Tv, utilizada en el canal Dtt Lavoro, italiano. El proyecto i-LAB [ILAB PROJECT ITA,. 2007] se concibió para la realización de un entorno de prueba interconectado para apoyar y guiar el desarrollo de los servicios, productos y aplicaciones interactivas basadas en el estándar MHP (Multimedia Home Platform), dentro del marco de desarrollo impulsado por el despliegue de la TDi y el Plan Nacional de I+D en España 2004-2007.La infraestructura de soporte esta diseminada en diferentes laboratorios especializados en universidades y centros tecnológicos españoles.

En Lopez-Nores et al. [2010a] se presenta una arquitectura de software basada en Java para el desarrollo aplicaciones y servicios de T-Learning. El marco propuesto proporciona apoyo adecuado para las necesidades específicas de esta área, con un fuerte énfasis en la interactividad, promoviendo la creación de comunidades de estudiantes y espacios de trabajo compartidos en una base peer-to-peer. De igual forma este proyecto aporta una herramienta CASE para el desarrollo visual de dichos servicios, distribuyendo su funcionalidad entre una serie de escenarios que prevén los roles diferenciados de una comunidad. Por otra parte Yun et al. [2011] muestran como IPTV ha dado un nuevo impulso al desarrollo y las formas de soportar la educación moderna, tomando relevancia la investigación en la construcción de un entorno de aprendizaje digital basado en IPTV. Su investigación se basa en un entorno IPTV abierto de aprendizaje digital, haciendo buen uso de ventajas como: las tecnologías de codificación, la función interactiva y la capacidad de transmisión de contenidos educativos a través de videos.

Lopez-Nores et al. [2010b] muestran como la implantación en el Espacio Europeo de la Educación Superior lleva a cabo una profunda renovación de las metodologías de enseñanza, haciendo hincapié en el papel de las TIC para permitir posibilidades de aprendizaje continuo y de por vida. Se describe un proyecto pionero en la aplicación de tecnologías de TDi a la educación superior, como medio para facilitar la comprensión de los conceptos que se enseñan en la facultad de Ingeniería de Telecomunicación de la Universidad de Vigo. Experiencias preliminares con estudiantes voluntarios revelaron el valor pedagógico y motivador del enfoque propuesto, dando observaciones útiles para la aplicación del paradigma de T-Learning.

Además existen otros proyectos que explotan las capacidades de la TDi en diferentes áreas como: el proyecto T-HEALTH, oficialmente llamado "Piloto para el estudio de las mejoras de los mecanismos de atención a pacientes crónicos mediante TDT", cuyo objetivo es el desarrollo de la infraestructura tecnológica para la atención de pacientes crónicos mediante TDT [Proyecto Uvigo, 2010, 2010]. El proyecto Redes Híbridas [PRH, 2008] que busca desarrollar una plataforma de gestión y distribución de contenidos multimedia turísticos, combinando las diferentes redes de transmisión y acceso a los contenidos (Internet, televisión digital y móvil) para ofertar contenidos avanzados a los turistas. El proyecto TDTD [Proyecto Uvigo, 2010] "TDT orientada al Turismo y al patrimonio histórico", cuyos objetivos son los de desarrollar una solución libre y ofrecer un conjunto de servicios piloto, orientados al turismo y la difusión del patrimonio histórico cultural, los cuales permitan crear una comunidad de administraciones locales y empresas que compartan experiencias y conocimiento en el desarrollo de servicios interactivos al ciudadano, utilizando para ello la TDT [Aarreniemi, 2006]. A diferencia de los proyectos aquí presentados, la contribución de este trabajo es la propuesta de una arquitectura para el soporte de CAV en ambientes de TDi, que permita el aprovechamiento de las ventajas de la interactividad, a través del consumo flexible y eficiente de servicios asociados o no a los contenidos transmitidos. Esta arquitectura tiene la capacidad de soportar el desarrollo de otros proyectos semejantes a los descritos en esta apartado, convirtiéndose en el punto de referencia para las investigaciones sobre TDi que se vienen desarrollando en Laboratorio de TDi de la Universidad del Cauca [Campo, 2009].

\section{MARCO TEÓRICO}

En este apartado se plantean los temas más relevantes en torno a: Redes Sociales, Arquitecturas de acceso a servicios, CAV, TDi y T-Learning; que corresponden a los elementos esenciales del marco teórico de esta investigación.

\section{Redes Sociales}

Gracias a la flexibilidad del protocolo IP, ha sido posible poner a disposición del mundo, múltiple cantidad de servicios, librando barreras geográficas o tecnológicas. Son muchos los servicios que han sido desplegados en Internet o "virtualizados", con el objetivo de hacer la vida más cómoda, tal es el caso de: correo electrónico, pagos o transferencias electrónicas, radio, televisión, comercio electrónico, mensajería instantánea, galerías multimedia, entre otros [Tonteri et al., 2011]. En los últimos años Internet ha tenido una evolución importante, al considerar de manera más amplia el aspecto social y enfocarse en explotar el potencial educativo de la red, así como fomentar la colaboración y el intercambio ágil de información entre los usuarios. Esta nueva tendencia es conocida como Web 2.0, a través de la cual han surgido nuevos 
conceptos de servicios, tales como: Blogs, Wikis, RSS (Really Simple Syndication), Tags; aplicaciones a tener en cuenta en labores educativas, considerando las ventajas para realizar procesos de gestión de contenidos [de la Torre, 2006]. Las Redes Sociales hacen uso de los Servicios de la Web 2.0, permitiendo el intercambio dinámico de información entre los usuarios y la integración de aplicaciones en una sola plataforma [Vidal et al., 2011]. Estos rompecabezas de aplicaciones se conocen como Mashups y permiten que una aplicación Web use recursos (aplicaciones ó servicios) de más de una fuente para crear servicios completos [Bond y Cheung, 2008]. Una de las tecnologías más apropiadas para la implementación de estos son los Servicios Web, en los cuales es posible la implementación y composición de servicios [Sabbouh et al., 2007]. Dentro de las implementaciones comunes para servicios Web, están las basadas en los protocolos SOAP (Simple Object Access Protocol) y REST (Representational State Transfer). El primero de estos, es usado para la comunicación entre cliente y servidor mediante el intercambio de mensajes basados en XML (WSDL), mientras que en el segundo, los mensajes son por lo general en formato JSON [Chanchí et al., 2011]. En ambos casos tanto el cliente como el servidor deben conocer el formato y lenguaje de los mensajes para poder encapsular y des-encapsular peticiones y respuestas. Dada la sencillez, flexibilidad y facilidad de procesamiento de los mensajes, el protocolo REST-JSON suele ser usado en la implementación de servicios para redes sociales.

\section{Arquitectura de Servicios - REST}

El protocolo REST, plantea un estilo de arquitectura cliente-servidor en la cual un servicio es visto como un recurso y es identificado a través de una URL, mediante la cual este puede ser consumido. Para acceder a los servicios Web, se hace uso de mensajes en formato simple, los cuales se intercambian entre cliente y servidor. REST define a partir del protocolo HTTP, cuatro métodos: GET, PUT, DELETE y POST, de los cuales los más usados son: GET y PUT. Para la comunicación e intercambio de información entre cliente y servidor a través de REST, se puede hacer uso de diversos formatos y lenguajes: XML, HTML, JSON; debido a la sencillez y facilidad de procesamiento de los mensajes JSON, este es el tipo de mensajes mas usado en servicios de redes sociales y comunidades en Internet. Cada mensaje intercambiado contiene la información necesaria para el funcionamiento de los servicios, de tal forma que para cada servicio el cliente y servidor conocen el protocolo interno de los mensajes. JSON es un formato ligero basado en texto, cuya sintaxis se basa en un subconjunto de funcionalidades y palabras reservadas del lenguaje JavaScript. Un mensaje JSON está constituido por dos estructuras básicas: la primera es una colección de parejas o estructuras nombre-valor, conocidas como: registros, diccionarios, hash, listas de claves o arreglos asociativos; y la segunda es una lista ordenada de valores, la cual suele representarse en la mayoría de los lenguajes en forma de: arreglos, vectores, listas, etc. Las anteriores estructuras son usadas para conformar los mensajes de intercambio. A continuación se presenta el esquema de consumo de servicios REST-JSON en entornos de TDi [Chanchí et al., 2011], ver Figura 1.

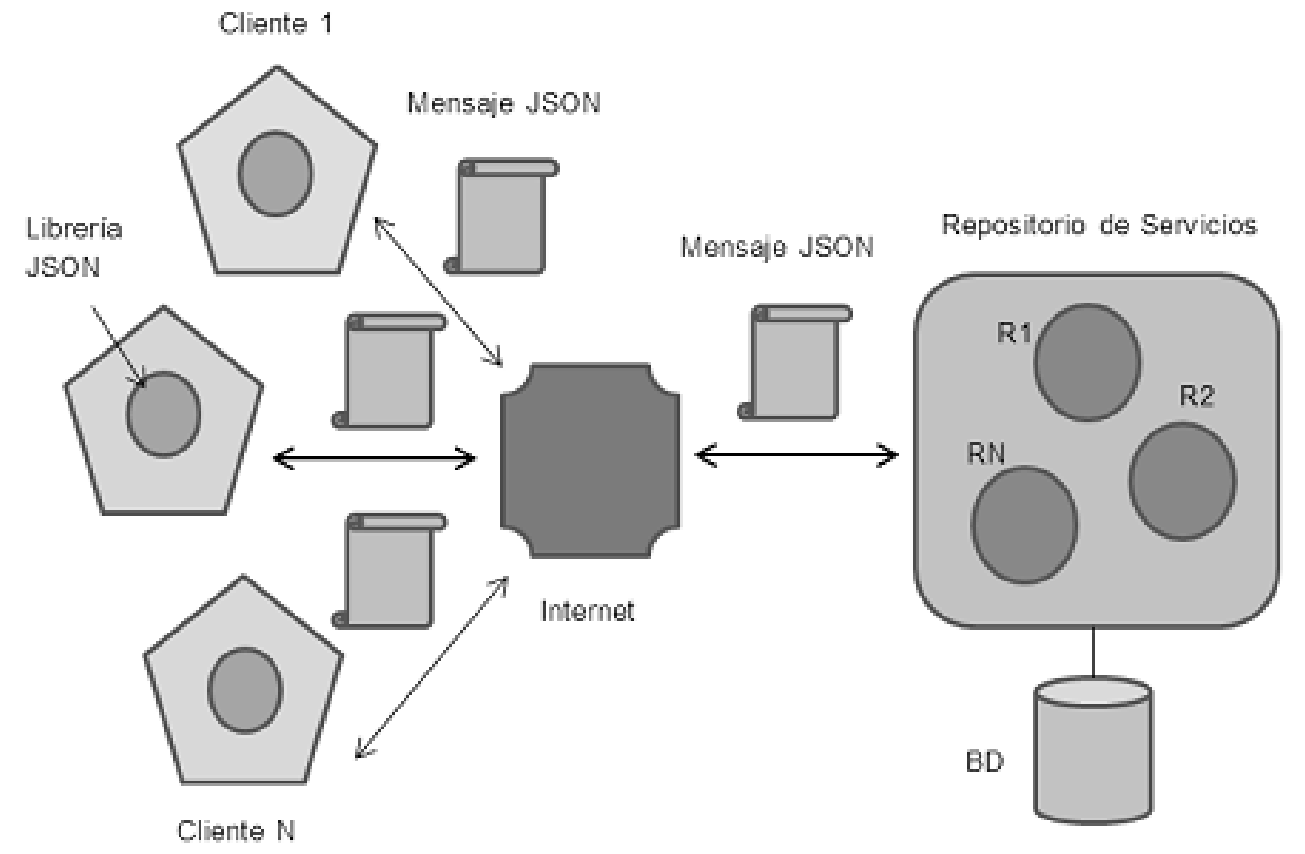

Fig. 1: Esquema de consumo de servicios REST-JSON 
Tal como se presenta en la Figura 1, cada servicio es representado como una instancia o recurso: $\mathrm{R} 1, \mathrm{R} 2, \ldots, \mathrm{RN}$, dentro del repositorio de servicios o servidor de aplicaciones; de tal forma que estos, están en la capacidad de interactuar entre sí para procesos de orquestación. A cada recurso o servicio se le asigna una URL desde la cual, los clientes: $1,2, \ldots, N$, pueden acceder vía Internet y recibir el mensaje correspondiente a cada recurso; para el caso del escenario de televisión el acceso a Internet es a través del canal de retorno. El mensaje recibido por cada cliente, contiene una estructura con un conjunto de parejas nombre-valor conocidas por los clientes y el servidor de aplicaciones, las cuales son des-encapsuladas por cada cliente, y de acuerdo al nombre del atributo, son clasificadas funcionalmente y presentadas en la interfaz correspondiente; cada tipo de cliente debe contar con la librería apropiada según las características de su Hardware, para permitir la interpretación de los mensajes JSON; de igual forma el cliente deben contar con las capacidades necesarias para hacer peticiones HTTP de tipo GET o PUT propias del protocolo REST. En la presente arquitectura es el Set-Top Box (STB) el encargado de realizar las peticiones e interpretar los mensajes de acuerdo al esquema REST - JSON [Chanchí et al., 2011].

\section{Comunidades Académicas Virtuales}

Una CAV se forma por uno o varios grupos de individuos que están vinculados por intereses en común, los cuales están comprometidos en un proceso de aprendizaje continuo, y su principal objetivo es el de construir conocimientos de forma compartida utilizando las TIC como un medio de expresión, como herramienta de comunicación, como recurso didáctico e incluso como instrumento de gestión [Gal-Oz et al., 2010]. Así, las CAV se consideran un recurso de gran demanda en el mundo, por ser conductoras de aprendizaje continuo y participes de la construcción del conocimiento. Actualmente estas comunidades han generado nuevos ambientes en el proceso de la enseñanza y aprendizaje, ya que potencializan los medios de comunicación que existen, para compartir información y al mismo tiempo conocimiento como lo demuestra el proyecto descrito en Mendiola [2008]. Además se cuenta con la comunicación a través de computadores sobre los cuales se basan las CAV, estableciendo una nueva pedagogía, que Haughey y Anderson [1998] llaman "pedagogía de Internet".

\section{Televisión Digital Interactiva}

La Televisión Digital interactiva (TDi) sobrepasa la televisión analógica en varios aspectos: capacidad, mejor aprovechamiento del espectro, mayor inmunidad al ruido o interferencias, mejor calidad de imagen y sonido, posibilidad de transmisión de datos simultáneamente, ahorro de potencia en la transmisión. Sin embargo, la principal ventaja está en la capacidad de interactuar con el usuario. La interactividad permite al usuario ser parte activa de la programación, brindando la posibilidad de consultar o extender la información presentada, combinar contenidos multimedia (audio, video, texto), participar en foros de opinión y además controlar de cierta manera la secuencia de la información presentada [Arciniegas et al., 2011]. La Figura 2 muestra una cadena convencional extremo a extremo de TDi donde se observan cuatro tipos de actores bien definidos: Proveedor de contenidos y servicios, Broadcaster, Operador de red y Usuario final. Esta cadena de TDi se encuentra descrita detalladamente en Campo et al. [2009].

El proveedor de contenidos es cualquier empresa que gestiona el contenido multimedia, lo proporciona para su distribución a través de la infraestructura de red de televisión, lo crea o adquiere con terceros, además autoriza quién puede ingresar, distribuir o compartirlo. El Broadcaster adapta y multiplexa los contenidos de audio y video en un flujo de transporte, se encarga también de generar e inyectar el carrusel de objetos que transporta las aplicaciones interactivas. El Operador de red adapta y difunde el flujo de transporte a través de una red de televisión digital ya sea satelital, terrestre o de cable, siguiendo las especificaciones del estándar de televisión digital. El Usuario final a través de un equipo terminal (televisor y/o STB), recibe y controla los contenidos y servicios. Además puede enviar y/o recibir información a través de un canal de retorno de vuelta hacia el proveedor de contenidos y servicios. (Campo et al., 2009).

\section{T-Learning}

El término T-Learning es definido como aprendizaje a través de televisión interactiva, lo cual hace referencia a tener acceso interactivo a materiales de aprendizaje ricos en video mediante un televisor u otro dispositivo que tenga más características de televisor que de computador. Dicho dispositivo tiene que ser confiable y fácil de utilizar [Dosi y Prario, 2004; Uvigo, 2010]. La educación virtual usa a menudo como apoyo las herramientas de Internet, sin embargo este escenario es una limitante, sobre todo en el caso de países en vía de desarrollo. En el mundo la penetración de la televisión es mucho mayor comparada con Internet, es por ello que se exploran nuevas posibilidades para hacer que la educación virtual se introduzca, utilizando ahora la televisión como medio para llegar a los estudiantes. Los proyectos en T-Learning buscan dar solución a algunos de los problemas de e-Learning con la ayuda de TDi. Por ejemplo Aarreniemi [2006] busca ampliar el acceso ofreciendo educación formal permanente, permitiendo la interacción en el 
aprendizaje vía TDi. Uno de los retos es adaptar y gestionar CAV soportadas por tecnologías como la TDi, que permitan tener una mayor penetración sobre las poblaciones donde Internet aún no es una opción.

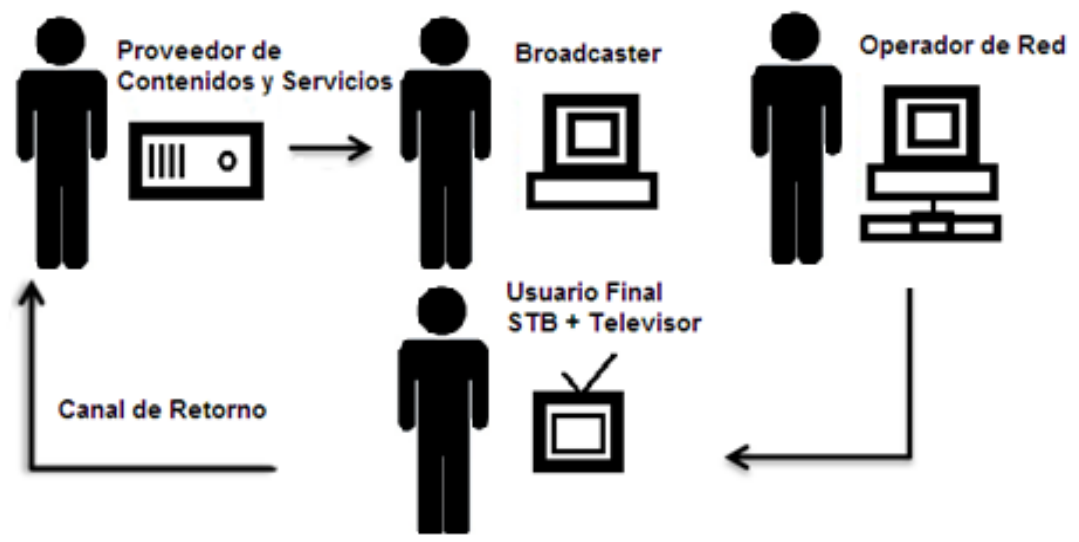

Fig. 2: Cadena extremo a extremo para TDi

\section{ARQUITECTURA PROPUESTA}

La arquitectura propuesta en este artículo busca potencializar la principal característica de la TDi (la interactividad), a través del acceso a servicios o aplicaciones tomadas de la Web 2.0, para el soporte de CAV en entornos de televisión. Para el acceso a estos servicios se hace uso del esquema REST-JSON el cual permite la integración de los escenarios de T-Learning e Internet. Esta arquitectura aprovecha la flexibilidad y escalabilidad de los servicios en la Web 2.0 (Foros, Chat, Gestión de Contenidos, trabajo colaborativo, entre otros), siendo consumidos de manera remota por parte del usuario usando el esquema REST-JSON propuesto por Chanchí et al. [2011]. Los servicios pueden ser asociados o no al contenido multimedia difundido y pueden ser accedidos mediante el canal de broadcast o el canal de retorno.

La Arquitectura propuesta (ver Figura 3) da solución a los siguientes escenarios de interacción: Escenario A: Servicios asociados al contenido que utilizan el canal de broadcast. En este escenario el televidente solicita un servicio, el cual está asociado al contenido trasmitido a través del canal de broadcast, por ejemplo el televidente está viendo un curso de hidrocarburos y desea más información sobre compuesto químico en particular; Escenario B: Servicios asociados al contenido que utilizan el canal de retorno. En este escenario el televidente solicita un servicio, el cual está asociado al contenido trasmitido a través del canal de broadcast, a diferencia del anterior estos servicios usualmente solicitan mayor información al usuario (mayor interacción), por ejemplo el televidente desea realizar una evaluación del curso; Escenario C: Servicios no asociados al contenido que utilizan el canal de broadcast. En este escenario el televidente solicita un servicio, que no está ligado al contenido, por ejemplo el televidente desea ver la guía de programación del día siguiente; y Escenario D: Servicios no asociados al contenido que utilizan el canal de retorno. En este escenario el televidente solicita un servicio independiente del contenido difundido, por ejemplo el televidente solicita configurar las preferencias de su perfil. La Figura 3 extiende la cadena convencional extremo a extremo de TDi, donde los actores definidos en la Figura 2 siguen siendo válidos en la arquitectura propuesta. A continuación se definen los componentes adicionales que se han introducido para el soporte de una CAV.

\section{Directorio de Servicios Virtuales}

Contiene la lista de servicios en conjunto con su descripción disponibles para ser accedidos desde la CAV. En este componente se registran los servicios que van a ser ofrecidos parte de los Proveedores de Servicios, utilizando para ello cadenas de caracteres con la sintaxis nombre-valor, sobre documentos JSON. Este grupo de cadenas corresponden a direcciones URL con la ubicación de los diversos servicios a ser consumidos; así, los metadatos almacenados sirven como mecanismos de búsqueda inteligente de servicios y composición u orquestación de estos.

\section{Proveedor de servicios}

Contiene los servicios de soporte para las CAV, tales como: Foros, Chats, Wikis, Sindicación, Información asociada al contenido y Gestión de Contenido. Estos servicios son desplegados como recursos, de acuerdo al concepto propuesto por el esquema de servicios REST-JSON y pueden ser desplegados a 
través de la Red en diversos servidores de aplicaciones. Los proveedores de servicios pueden desarrollar las aplicaciones interactivas bajo estándares como MHP, las cuales son necesarias para ser agregadas al contenido multimedia. En el caso de TDi, el canal de broadcast es limitado al proveer solo interactividad local, es por ello necesario agregar un canal de retorno, que permita la interactividad y el consumo de servicios por ese medio; por lo general ese canal de retorno suele ser de tipo IP [ITU-T. 2007]. Los servicios desplegados en este componente pueden ser asociados o no al contenido y ser consumidos desde el canal de broadcast o a través del canal de retorno. Los servicios consumidos por el canal de broadcast son enviados a través del carrusel de objetos propio del estándar de TDi, mientras que a través del canal de retorno los servicios son consumidos siguiendo el esquema de servicios Web REST-JSON. Mediante este esquema se hacen peticiones HTTP desde el STB hacia los servidores de aplicaciones y se procesan las respuestas obtenidas en formato JSON.

\section{Preferencias y perfil de usuario}

Es el encargado de manejar las preferencias y perfiles de usuario. Esta información es almacenada por primera vez cuando el usuario se registra vía Web a la CAV y es actualizada de manera constante a medida que el usuario participa en la comunidad generando y valorando el contenido; opcionalmente la información del perfil de usuario puede ser modificada por el usuario a través de la Web siguiendo algunas políticas de seguridad. Es importante destacar que este componente almacena también las características de presentación de los servicios, tales como: tipo y tamaño de fuente, colores, disposición de los elementos en pantalla, diseño del perfil dentro de la comunidad, entre otros. Este bloque funcional esta soportado en una ontología de preferencias o perfiles, definida y adecuada de acuerdo al ámbito de la CAV en cuestión y al modelo funcional del proyecto ST-CAV.

\section{Mediador}

El componente mediador se encarga de recibir las solicitudes o peticiones del usuario a través del canal de retorno y de sincronizar el canal de broadcast y el canal de retorno. Para el caso de los servicios asociados al contenido, las aplicaciones son indexadas a un contenido en específico. Este proceso de asociación se hace mediante el manejo de documentos en formato JSON, los cuales son accedidos por el Mux de Contenidos y Servicios para realizar la integración por el canal de broadcast. En el caso de que los servicios y aplicaciones vayan por el canal de retorno, es el sistema mediador el encargado de coordinar al Directorio de Servicios Virtuales, para que entregue los servicios o aplicaciones al usuario mediante el protocolo REST y en el formato de los documentos JSON. Además, el mediador realiza procesos de sincronización de las aplicaciones y servicios con el canal de broadcast. Así mediante este módulo se logra dotar de flexibilidad a la arquitectura, al permitir el manejo de contenidos por el canal de broadcast y el canal de retorno asociados y no asociados al contenido.

\section{Canal de Retorno}

Consiste en una conexión bidireccional entre el usuario y sistema mediador, es utilizado para hacer peticiones (en este caso de tipo HTTP REST) o responder al proveedor de contenidos, también para enviar respuestas hacia el STB (en formato JSON) y cualquier otra comunicación necesaria para la prestación de servicios interactivos. Así, de acuerdo a como se utilice el canal de retorno la interactividad se puede clasificar en interactividad local e interactividad remota: i) Interactividad Local: la información es enviada mediante el flujo broadcast (usando el carrusel de objetos) y el televidente puede interactuar localmente con la información almacenada en el dispositivo; e ii) Interactividad remota: el usuario interactúa con el proveedor de contenidos remoto, al que se conecta mediante un canal de retorno. En este último caso las respuestas son enviadas usando el protocolo REST, mediante mensajes JSON, el cliente (STB) se encarga de interpretar los mensajes y presentar la información en la pantalla del televisor.

\section{Mux de Contenidos y Servicios}

Es quien permite la programación de la emisión y es el encargado de generar un flujo de transporte (programa de televisión) mediante la multiplexación o integración, de los contenidos de televisión con las aplicaciones o servicios e información de control y sincronismo; generando el carrusel de objetos. Las aplicaciones son embebidas sobre el contenido mediante DSM-CC (Digital Storage Media-Command and Control), el cual es un estándar de difusión de datos que permite tanto transmitir aplicaciones y datos MHP vía un canal de broadcast en forma de carrusel, como sincronizar estas aplicaciones con el audio y el video de un servicio mediante los llamados flujos de eventos. 


\section{Codificador-Modulador}

El codificador se encarga de dar una altísima protección al flujo de transporte, tal que permita la correcta transmisión a través de los diferentes medios (satelital, terrestre o cable). El Modulador, realiza la adaptación de los flujos de televisión a una señal adecuada para la transmisión bajo un estándar determinado para televisión.

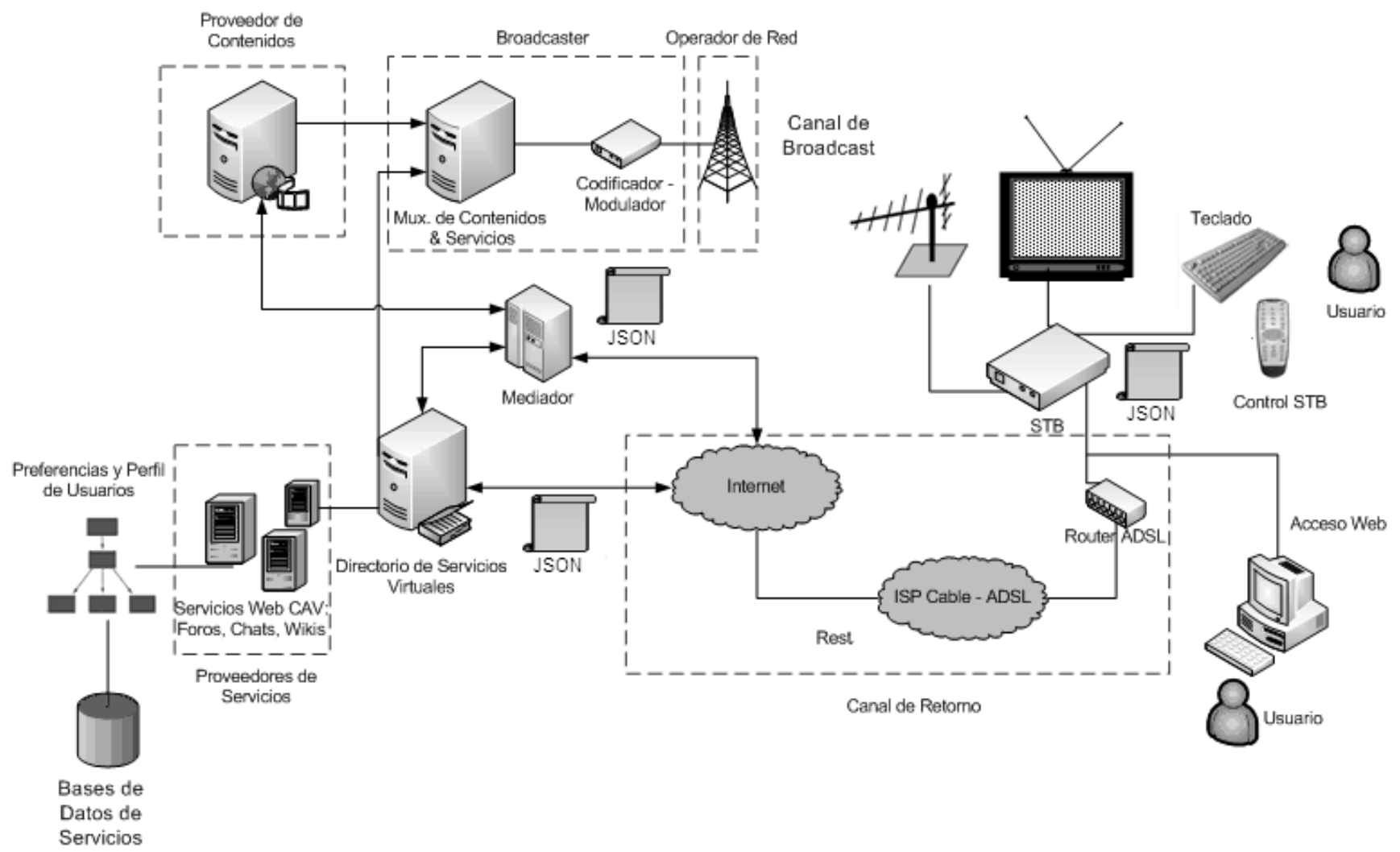

Fig. 3: Arquitectura Propuesta

\section{Set-Top Box (STB)}

En la arquitectura propuesta el televisor es un elemento de despliegue de información. Siendo el STB el encargado de manejar la interactividad a nivel de usuario, pues es el dispositivo que recibe las peticiones o eventos del usuario y las envía a través del canal de retorno pasando por el mediador hasta el directorio de servicios. Las peticiones son generadas por el STB y tienen el formato de los mensajes HTTP (GET y PUT) del protocolo REST, razón por la cual, el STB debe permitir el soporte de librerías para el procesamiento de los mensajes de respuesta en formato JSON. Otra de las funciones que cumple el STB es determinar la disposición y las características gráficas de los servicios en la pantalla del televisor (colores, tipos de fuente, etc); para ello es necesario tener en cuenta las recomendaciones para despliegue de contenidos de TDi propuestas en Campo et al. [2010b]. De estas recomendaciones se destaca la referente a la ubicación de los servicios a la derecha de la pantalla, dándole mayor importancia al contenido multimedia.

Además de lo anterior es importante tener en cuenta, que la mayoría de los servicios de las CAV, requieren el uso de texto para su interacción (Chat, Foros, Wikis, entre otros), es por ello recomendable que el STB cuente con interfaces opcionales que le permitan conectarse con otros dispositivos que faciliten la interacción como por ejemplo, un teclado, micrófono, controles remoto adicionales, entre otros.

\section{RESULTADOS}

La arquitectura propuesta está enmarcada dentro del proceso de investigación del T-Learning, que se viene desarrollando en la Universidad del Cauca (Colombia) con la cooperación de la Universidad de Oviedo (España) y la Universidad Autónoma de Occidente (Colombia), a través de los proyectos EDiTV [Arciniegas et al., 2011], SOLITE [Acevedo et al., 2010] y ST-CAV [Acevedo et al, 2010]. Actualmente, parte de la arquitectura propuesta se ha implementado dentro del laboratorio experimental de TDi de la Universidad del Cauca. En Colombia y Latinoamérica el proyecto EDITV fue pionero en este campo donde los resultados más destacados son: una arquitectura de laboratorio experimental para TDi bajo el estándar DVB-C [Campo et al., 2009], Análisis de tráfico para un servicio de vídeo bajo demanda sobre redes HFC usando el 
protocolo RTMP [Campo et al., 2010a], Análisis del protocolo DOCSIS para la distribución de contenidos educativos de iTV en el canal interactivo de la red HFC [Campo et al., 2009], recomendaciones para el despliegue de contenidos de T-Learning [Campo et al., 2010b], una arquitectura básica de un navegador DVB-HTML para múltiples terminales [Cerón et al., 2009], directrices para el soporte de comunidades académicas virtuales en entornos de TDi [Chanchí et al., 2010] y Evaluación de la QoS en redes HFC para la distribución de aplicaciones de televisión digital interactiva (TDI) [Rueda et al., 2012].

La arquitectura básica de un navegador DVB-HTML para múltiples terminales fue pensada para permitir a personas interactuar con aplicaciones DVB-HTML con fines complementarios a la educación virtual basada en computador. El usuario debe tener acceso al sistema de difusión de televisión digital, en el cual se emiten aplicaciones y contenidos educativos. La arquitectura del navegador está dividida en tres componentes relacionados con: el acceso al canal de broadcast y canal de retorno; el procesamiento de información de los documentos de la aplicación y a la presentación y manejo de interfaces por parte de las aplicaciones de cada uno de los dispositivos [Cerón et al, 2009]. Además se generó contenidos para ser desplegados sobre dicho laboratorio. En la Figura 4, se ilustra la interfaz de uno de los cursos desplegados denominado AgroEDiTV biocombustibles como material de apoyo en el programa de Tecnología de Agroindustria de la Universidad del Cauca, lo cual permitió la validación de las recomendaciones para la generación y distribución de contenidos educativos orientados a TDi (Campo et al, 2010b).

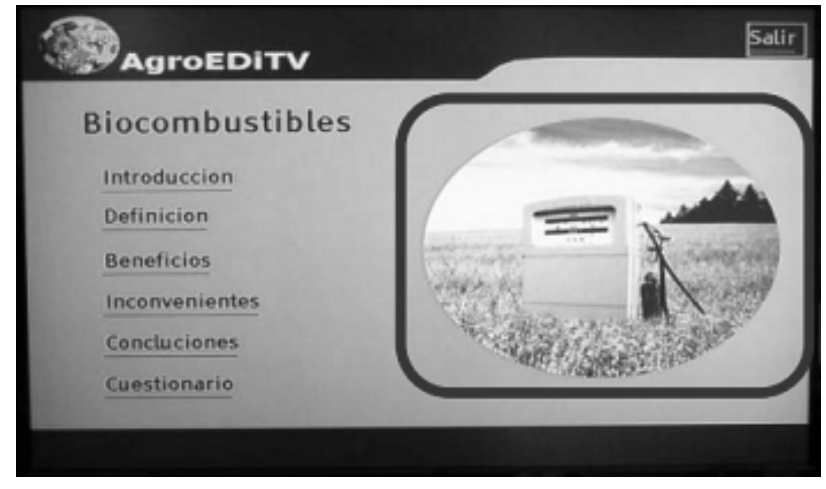

Fig. 4: Imagen curso AgroEDiTV

Además, se construyo una aplicación denominada Curso Piloto, la cual fue desarrollada bajo DVB-J (DVBJava) siguiendo las restricciones de MHP. Es una aplicación que permite navegar a través de los diferentes contenidos del curso e interactuar en diferentes etapas de este a través del control remoto del usuario y de la conexión IP con el servidor de Proveedor de Contenidos (ver Figura 5). Usando el canal de retorno para la solicitud de información tal que las aplicaciones están embebidas sobre el canal de broadcast.

Actualmente dentro del laboratorio experimental de Televisión Digital interactiva, se viene desarrollando el proyecto ST-CAV (Servicios de T-Learning para el soporte de comunidades académicas virtuales) [Proyecto ST-CAV, 2010], cuyo objetivo es dar soporte a una CAV en televisión desde diversos escenarios, tales como: Televisión Digital Terrestre, Televisión Móvil e IPTV; de esta forma, uno de los principales objetivos del proyecto ST-CAV es apoyar procesos de aprendizaje en televisión (T-Learning) a través de servicios de la Web 2.0 (Chat, Foros, Blogs, Wikis, entre otros), buscando la construcción de conocimiento entorno a diversas temáticas de una CAV. A modo de piloto inicial, en el proyecto ST-CAV se implementaron los servicios de tablón de mensajes y sala de chat, ver Figura 6. El objetivo de estos servicios es permitir la interacción de los miembros de una comunidad en torno a un contenido multimedia, de tal forma que se puedan expresar opiniones y discusiones respecto a una temática de interés.

El servicio de tablón de mensajes o micro-blog, ver Figura 6, consiste en un mini foro, similar al usado en la red social Twitter, en el cual los miembros de una CAV pueden publicar mensajes y comentarios con un número restringido de caracteres, mientras están viendo un programa de televisión. La interfaz del servicio de tablón de mensajes permite listar los últimos 5 mensajes de los miembros de la comunidad y navegar a través de ellos mediante las flechas del control. El servicio de sala de chat por su parte, sigue el mismo principio del micro-blog, de tal modo que cada miembro de la comunidad puede expresar una idea al grupo de miembros de la comunidad. Una de las principales características que debe tener el contenido multimedia es la de promover la participación a través de estos servicios, dado que de lo contrario puede dispersar la atención del televidente. Los mensajes en formato JSON que se intercambian en los servicios, constan de un conjunto de parejas nombre-valor (userid, mensaje, estampa de tiempo), las cuales contienen el identificador de usuario, la idea publicar y un dato numérico que representa la estampa de tiempo a partir de la cual se obtiene la fecha de la publicación. EI STB se encarga de procesar el mensaje 
JSON, obteniendo cada uno de los valores de las parejas y presentando la información del servicio en la pestaña correspondiente. Es importante tener en cuenta, que la información presentada en la pantalla del televisor debe seguir las recomendaciones para despliegue de contenido en TDi [Campo et al., 2010b], de las cuales se destacan las siguientes: el tipo de letra usada para las aplicaciones y los contenidos debe ser Tiresias, con un tamaño mínimo de 18 puntos; los servicios deben ser dispuestos en la parte derecha de la pantalla, mientras el contenido multimedia debe ser ubicado a la izquierda; el número de palabras por cada pantalla debe ser como máximo de 90.

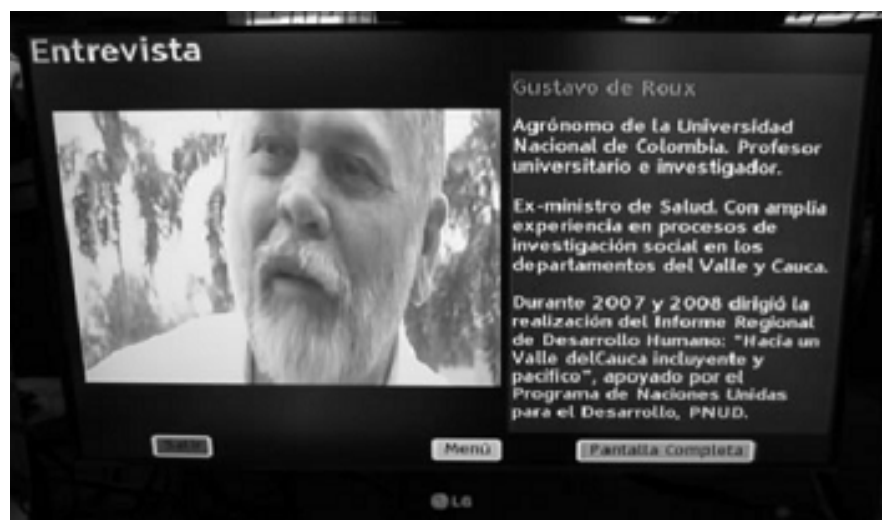

Fig. 5: Imagen Curso Piloto

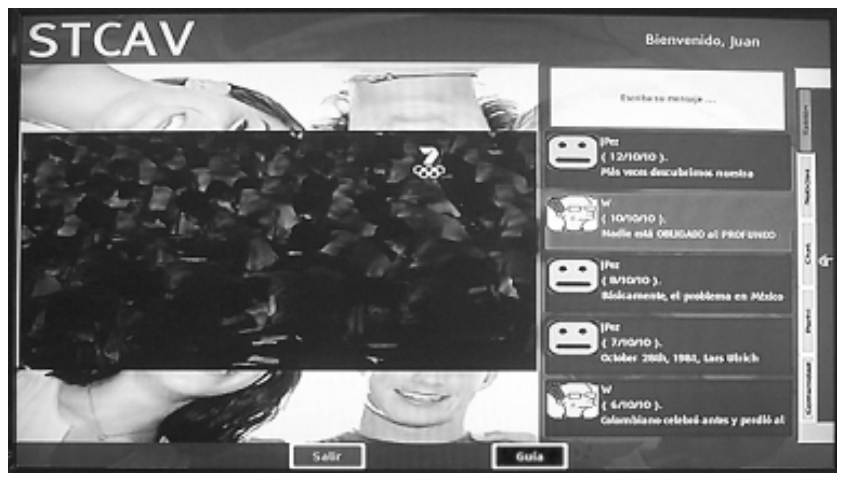

Fig. 6: Piloto del Proyecto ST-CAV

Así, la validación de la arquitectura propuesta es un proceso incremental que recoge como punto de partida los resultados y las implementaciones de aplicaciones reales implementadas en el laboratorio montado a partir de la arquitectura propuesta. La arquitectura presentada en este artículo no sólo se limita al soporte de aplicaciones interactivas, sino que busca cumplir con uno de los objetivos del proyecto ST-CAV dando soporte a los servicios de una comunidad académica virtual en ambientes de TDi. Es importante destacar dentro de la arquitectura la flexibilidad para soportar servicios asociados y no asociados al contenido, tanto a través del canal de broadcast, como del canal de retorno, estos últimos con más interactividad que los primeros. De igual manera cabe anotar que estos servicios tienen características de distribución y escalabilidad, teniendo en cuenta las ventajas de tecnologías como los Servicios Web basados en el esquema REST-JSON, permitiendo el uso de los mismos a futuras implementaciones o en diversos ambientes de Televisión (Móvil, IPTV). Finalmente, resaltar el manejo de la sincronización dentro de la presente arquitectura, entre el canal de broadcast y el canal de retorno, componente a ser tenido en cuenta en proyectos que pretenden dotar a las telecomunicaciones de las ventajas que provee Internet.

\section{CONCLUSIONES}

La arquitectura para el soporte de comunidades académicas virtuales - CAV en ambientes de TDi, provee aportes importantes en el área de la telemática, tales como: el despliegue de comunidades académicas virtuales en un escenario diferente a Internet; la integración de servicios de Internet (Web 2.0) en ambientes de Telecomunicaciones mediante el esquemas de consumo de servicios REST-JSON; el soporte para interactividad a través del canal de retorno IP y la difusión de la TDi como tecnología llamada a ampliar el abanico de oportunidades para el soporte de educación virtual. Además de lo anterior, cabe resaltar que la flexibilidad en el despliegue y consumo de servicios aportado por la presente arquitectura puede ser adoptado en otros escenarios de televisión como IPTV y TV Móvil. 


\section{AGRADECIMIENTOS}

Este trabajo ha contado con el apoyo de los proyectos MT2TDi financiado por la Vicerrectoría de Investigaciones de la Universidad del Cauca, SOLITE financiado por programa CYTED, ST-CAV financiado por Colciencias y el SENA. De igual manera este trabajo ha sido apoyado por el programa de Formación de Doctorados Nacionales de Colciencias, Convocatoria $N^{\circ} 528$.

\section{REFERENCIAS}

Aarreniemi, P., Modeling and Content Production of Distance Learning concept for Interactive Digital Television. Tesis Doctoral. Universidad de Tecnología de Helsinki, Finlandia. http://lib.tkk.fi/Diss/2006/isbn9512285428/ (2006).

Acevedo, C., Arciniegas, J., García X. y Perrinet, J., Proceso de Adaptación de una Aplicación de e-aprendizaje a t-aprendizaje, Revista Información Tecnológica. tecnol. v.21 n.6 La Serena 2010, Información Tecnológica Vol. 21(6), 27-36 (2010).

Alic, K., Zajc, M., Tkalcic, M., Burnik, U. y Tasic, J., Development of interactive television t-learning course, in Electrotechnical Conference, 2008. MELECON. The 14th IEEE Mediterranean, pp. 139 -144 (2008).

Arciniegas, J., Amaya, J., Urbano, F., Campo, W. Euscategui, R., García, A. y García, X., 2008, EDiTV: Educación virtual basada en televisión interactiva para soportar programas a distancia. E-Colabora: Revista De Ciencia, Educación, Innovación Y Cultura Apoyadas Por Redes De Tecnología Avanzada. v.1 fasc.1 p. $42-47$ (2011).

Ashwin, P., Changing Higher Education: The Development of Learning and Teaching, $1^{\text {a }}$ ed., pag. 79, Routledge Pub., Taylor and Francis, New York, USA (2006).

Bond, G. y Cheung, E., A Framework for Converged Telecom Services and Mashups. AT\&T Labs Research Florham Park, NJ, USA. http://echarts.org/Downloads/View-document/A-Framework-for-ConvergedTelecom-Services-and-Mashups-2008.html (2007).

Campo, W. Y., Arciniegas, J., García, R. y Melendi, D., Análisis de tráfico para un servicio de vídeo bajo demanda sobre redes HFC usando el protocolo RTMP. Revista Información Tecnológica, 2010, 21 (6) (2010a).

Campo, W. Y., Chanchí, G. E. y Urbano, F., Recomendaciones para el despliegue de contenidos de TLearning. XI congreso internacional de interacción persona ordenador, Valencia, España (2010b).

Campo, W. Y., Rueda, D. F., Taimal, I. R. y Arciniegas, J., Análisis del protocolo DOCSIS para la distribución de contenidos educativos de iTV en el canal interactivo de la red HFC. Revista Avances en Sistemas e Informática, Universidad Nacional de Colombia Sede Medellín (2009).

Cerón, R., Martínez, F. y Castillo, J., Arquitectura Base de un Navegador DVB-HTML para Múltiples Plataformas. Tendencias, pp. 100-105. Cuenca, Ecuador (2009).

Chanchí, G. E., Campo, W. Y. y Arciniegas, J., Directrices para el soporte de comunidades académicas virtuales en entornos de TDi. 15 Convención Científica de Ingeniería y Arquitectura (15 CCIA), La Habana, Cuba (2010a).

Chanchí, G. E., Campo, W. Amaya, J. Y. y Arciniegas, J., Esquema de Servicios para Televisión Digital Interactiva, basados en el protocolo REST-JSON. Cadernos de Informática, vol. 6, n. ${ }^{\circledR}$ 1, pp. 233-240 (2011).

Dosi, A. y Prario, B., New Frontiers of T-Learning: the Emergence of Interactive Digital Broadcasting Learning Services in Europe, Proceedings of World Conference on Educational Multimedia, Hypermedia \& Telecommunications, ED-Media 2004, 4831-4836 (2004).

eLearning WORKSHOPS [En línea] http://www.elearningworkshops.com/modules.php?name=infocva Acceso: 12 de Noviembre de 2012.

Haughey, N. y Anderson, T., Networked learning: the pedagogy of the INTERNET. Chenelière/McGraw Hill. Toronto, Canadá (1998). 
ILAB PROJECT ITA [En línea], Instituto Tecnológico de Aragón. http://ilab.ita.es/ Acceso: 12 de Noviembre (2012).

ITU-T., FG IPTV-DOC-0199 IPTV vocabulary of terms. Focus Group On IPTV (2007).

Lago, o., Farenhorst, R., Avgeriou, P., de Boer, R. C., Clerc, V., Jansen, A. y H. van Vliet., The GRIFFIN Collaborative Virtual Community for Architectural Knowledge Management, in Collaborative Software Engineering, I. Mistrík, J. Grundy, A. Hoek, y J. Whitehead, Eds. Springer Berlin Heidelberg, pp. 195-217 (2010).

Lopez-Nores, M., Blanco-Fernandez, Y. y Pazos-Arias, J., Architecting multimedia-rich collaborative learning services over Interactive Digital TV, in 2010 IEEE 5th Iberian Conference on Information Systems and Technologies (CISTI), pp. $1-6$ (2010a).

Lopez-Nores, M., Blanco-Fernandez, Y., Pazos-Arias, J. y Garcia-Duque, J., T-learning in Telecommunication Engineering: The Value of Interactive Digital TV in the European Higher Education Area, in 2010 IEEE 10th International Conference on Advanced Learning Technologies (ICALT), pp. 624 -626 (2010b).

Mendiola, M., Comunidades Académicas Virtuales como base para la construcción de conocimiento Casos: EDUSOL y Moodle Moot Perú, ESIME Culhuacan, Perú (2008).

Gal-Oz, N., Grinshpoun, T. y Gudes, E., Sharing Reputation Across Virtual Communities, Journal of Theoretical and Applied Electronic Commerce Research, VOL 5, AUGUST (2010).

Proyecto Uvigo [En línea], Universidad de Vigo. http://tvdi.det.uvigo.es/proyectos Acceso: 12 de Noviembre (2012).

PRH, Proyecto Redes Hibridas. [En línea] http://merlin.germinus.com/web/redeshibridas/inicio Acceso: 4 de Diciembre (2010).

Redondo, R., Vilas, A., Lopez, M., Arias, J., Solla, A. y Fernandez, Y., T-MAESTRO: Personalized learning for IDTV. Consumer Electronics, 2008. ISCE 2008. IEEE International Symposium on (2008).

Rueda, D., Campo, W., Taimal, I., Arciniegas, J., Evaluación de la QoS en redes HFC para la distribución de aplicaciones de televisión digital interactiva (TDI). Rev. Fac. Ing. Univ. Antioquia N. 63 pp. 9-19. Junio (2012).

Sabbouh, M., Higginson, J., Semy, Salim., Gagne, Dany., [En línea] Web Mashup Scripting Language. The Mitre Corporation. http://citeseerx.ist.psu.edu/viewdoc/download?doi=10.1.1.82.156\&rep=rep1\&type=pdf Acceso: 4 de Diciembre (2010).

Sancin, C., Castello, V., Dell Aiuto, V., Di Genova, D., T-learning for social inclusion. eLearning Papers www.elearningpapers.eu (2009).

Schröder, A., y Hölzle, K., Virtual Communities for Innovation: Influence Factors and Impact on Company Innovation, Creativity and Innovation Management, vol. 19, n. ${ }^{\circ}$ 3, pp. 257-268 (2010).

Tonteri, L., Kosonen, M., Ellonen, H., yTarkiainen, A., Antecedents of an experienced sense of virtual community, Computers in Human Behavior, vol. 27, n. ${ }^{\circ}$ 6, pp. 2215-2223. Noviembre (2011).

Vidal, C. E., Martínez, J. G., Fortuño, M. L., Cervera, M. G., Actitudes y expectativas del uso educativo de las redes sociales en los alumnos universitarios, RU\&SC. Revista de Universidad y Sociedad del Conocimiento, n. ${ }^{\circ}$ 1, pp. 171-185, (2011).

Yun, X. y Xiaozhe, M., Application of IPTV based on digital learning environment, International Conference on $\mathrm{E}$-Business and E -Government (ICEE), IEEE Xplore, pp. 1 -4 (2011). 